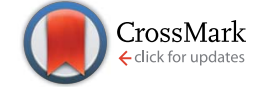

Cite this: RSC Adv., 2017, 7, 8241
Received 17th November 2016 Accepted 18th January 2017

DOI: 10.1039/c6ra26959a

www.rsc.org/advances

\section{Dietary intake of magnesium-L-threonate alleviates memory deficits induced by developmental lead exposure in rats}

\author{
Zhi-Yi Lou, ${ }^{a}$ Weiheng Chen, ${ }^{\text {b }}$ Wei-zhen Xue, ${ }^{a}$ Jin-Jun Ding, ${ }^{a}$ Qian-Qian Yang ${ }^{a}$ \\ and Hui-Li Wang*a
}

\begin{abstract}
Elevation of brain magnesium enhances cognitive capacity. Chronic lead (Pb) exposure has been implicated in the impairment of cognition. Whether elevation of brain magnesium rescues the impairment of memory by chronic $\mathrm{Pb}$ exposure is still unclear. Here we investigated the effect and possible mechanisms of chronic dietary intake of magnesium-L-threonate (MgT) on impaired spatial memory by chronic developmental $\mathrm{Pb}$ exposure in rats. We found that MgT rescued impaired spatial memory ability in $\mathrm{Pb}$-exposed rats. This positive effect is relative to increasing dendritic spine density and the expression of NR2A and GluR1.
\end{abstract}

\section{Introduction}

Lead $(\mathrm{Pb})$ is one of the most widespread and potential neurotoxins in the environment. Early developmental $\mathrm{Pb}$ exposure results in cognitive deficits, especially learning and memory, which can even last into adulthood despite the removal of $\mathrm{Pb}$ exposure..$^{1-4}$ There are many reports showing that chronic $\mathrm{Pb}$ exposure impairs hippocampal long-term potentiation (LTP), which is the cellular model of learning and memory. ${ }^{5-7}$ LTP is usually accompanied by spine formation and enlargement, ${ }^{8}$ Our previous work show that $\mathrm{Pb}$ exposure exerts a decrease in spine density, as well as the mushroom shaped spines in hippocampal pyramidal neurons in rats. ${ }^{9}$

In the mammalian brain, excitatory synapses are mainly localized in dendritic spines that express postsynaptic density (PSD), which include a major component, the PSD-95 family of proteins. To anchor and stabilize $N$-methyl-D-aspartate receptor (NMDAR) complexes at synaptic sites, some of the NR1 splice variants and NR2 subunits of NMDARs directly interact with the PSD-95 family of proteins. ${ }^{10} \mathrm{~Pb}$ exposure also inhibits the expression of the NR1 and NR2A subunit gene of NMDARs ${ }^{4}$ which is necessary for the induction of LTP. ${ }^{8}$

According to these, $\mathrm{Pb}$-induced cognitive deficits are related to impaired LTP, which are partly due to the reduction of dendritic spine density and the expression of some proteins anchored to PSD.

$\mathrm{Mg}^{2+}$ is one of the most abundant cations found in the body, and there are a lot of functions of which found in cells, including

${ }^{a}$ School of Food Science and Engineering, Hefei University of Technology, Hefei, Anhui 230009, PR China. E-mail: wanghl@hfut.edu.cn; Fax: +86 551 62919397; Tel: +86 551 62919397

${ }^{b}$ School of Life Sciences, University of Science and Technology of China, Hefei, Anhui 230026, PR China.E-mail: erwei@mail.ustc.edu.cn serving as a cofactor of enzymes, participating in the process of intracellular signal transduction as well as DNA and protein synthesis. In the central nervous system, $\mathrm{Mg}^{2+}$ plays an important role in neurotransmission, ${ }^{11}$ especially as a "gating" ion within the NMDAR complex ${ }^{\mathbf{1 2 , 1 3}}$ and the process of learning and memory. ${ }^{\mathbf{1 4 , 1 5}}$

Many in vitro studies show that increasing $\mathrm{Mg}^{2+}$ in neuronal cell cultures reduces oxidative cell death ${ }^{\mathbf{1 6}}$ and enhances the synaptic plasticity of cultured hippocampal neurons. ${ }^{17}$ By using a newly developed $\mathrm{Mg}^{2+}$ compound-magnesium-L-threonate (MgT) to elevate brain $\mathrm{Mg}^{2+}$, Slutsky et al. find it leading to significant enhancement of spatial and associative memory in rats. ${ }^{17}$ At the cellular level, $\mathrm{Mg}^{2+}$ increases the presynaptic boutons and enhances the protein level of NR2B-containing NMDARs in hippocampus thus resulting in enhancement of NMDAR signaling and synaptic plasticity. Furthermore, the effects of MgT on the protein expression of NR2B and brain derived neurotrophic factor (BDNF) are regional-special. All of these proteins are dependent on NMDAR signaling and leading to the enhancement of synaptic plasticity when elevated or activated. ${ }^{18}$ Based on the studies above, we hypothesized that MgT may rescue the impaired learning and memory ability induced by $\mathrm{Pb}$ exposure through the NMDA receptor.

In this study, we performed the experiments to raise the brain $\mathrm{Mg}^{2+}$ concentration to evaluate the effect of $\mathrm{MgT}$ on impaired learning and memory by chronic $\mathrm{Pb}$ exposure and to investigate whether the effect is related to spine density and the gene expression of NR2A, NR2B, GluR1 and PSD-95. MgT can be a potential complementary agent in the treatment of $\mathrm{Pb}$ intoxication.

\section{Materials and methods}

\subsection{Experimental animals and treatments}

Sprague-Dawley (SD) rats were obtained from the Laboratory Animal Center, Anhui Medical University, China. The Pregnant 
SD rats were randomly divided into two groups: control and $\mathrm{Pb}$ exposed group, drinking distilled water and $\mathrm{Pb}$ water $(300 \mathrm{ppm}$ $\mathrm{Pb}$ acetate in distilled water, $30 \mathrm{~mL}$ per day), respectively. The $\mathrm{Pb}$ exposed pups then acquired $\mathrm{Pb}$ indirectly via their mother's milk during lactation period. After weaning until the postnatal day 30 (PND30), the Pb-exposed pups had access to the same solution as their mothers. At the PND31, male rats of control were randomly divided into two groups: control and MgT group, while male $\mathrm{Pb}$ exposed group rats were randomly divided into two groups: $\mathrm{Pb}$ and $\mathrm{Pb}+\mathrm{MgT}$ group. The $\mathrm{MgT}$ and $\mathrm{Pb}+\mathrm{MgT}$ group received $\mathrm{MgT}$ for 30 days, while rats of control group and $\mathrm{Pb}$ group drank distilled water. The design of the experiment is shown in Fig. 1. All the animals were maintained on a $12: 12$ light/dark cycle in an air-conditioned constant temperature $\left(24 \pm 1^{\circ} \mathrm{C}\right)$ room. At the age of 61-67 days, animals were carried out the Morris Water Maze (MWM) test or other experiments. All animal experiments were performed following the guidelines of the National Institutes of Health Guide for the Care and Use of Laboratory Animals, and were approved by the Institutional Animal Care and Use Committee of Hefei University of Technology, China.

\subsection{Materials}

Magnesium-L-threonate powder (brand name Magtein ${ }^{\mathrm{TM}}$; MgT) was obtained from Center for Learning and Memory, School of Medicine, Tsinghua University, and its chemical structure is shown in Fig. 2. The protocol was adopted as that described previously. ${ }^{18,19}$ Simply, MgT was mixed in the rats' drinking water, and the concentration of MgT liquids was adjusted according to body weight measured every 3 days and the water intake on daily basis. Doing like these is to get as close as possible to the target dosage of $604 \mathrm{mg}$ per kg per day (50 mg per $\mathrm{kg}$ per day elemental magnesium), for this doses and time courses of MgT exposure have been shown to be effective in elevating brain magnesium and enhancing hippocampaldependent learning and memory in rats. ${ }^{19}$

\subsection{Tissue collection}

At the age of 70 days old, rat pups were deeply anesthetized with $\mathrm{CO}_{2}$ and decapitated. Brains were then removed from the skull quickly within $1 \mathrm{~min}$. Some brains were longitudinally cut into two halves; the left hemibrain was used for Golgi-Cox assay, while the right part was frozen and stored at $-80{ }^{\circ} \mathrm{C}$ for examining special protein expression.

\subsection{Pb determination in hippocampus}

$\mathrm{Pb}$ concentration in the hippocampus was estimated on the animals which had been used for behavioral experiment. After

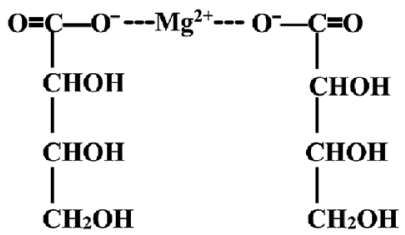

Fig. 2 The chemical structure of MgT.

decapitation of the animals, the hippocampus were isolated and digested with nitric acid. The $\mathrm{Pb}$ in the hippocampus was measured by Inductively Coupled Plasma Atomic Emission Spectrometer (CS600, LECO, USA) in Instruments' Center for Physical Science, University of Science \& Technology of China.

\subsection{Morris water maze test}

For assessing the effect of $\mathrm{MgT}$ and $\mathrm{Pb}$ on spatial memory ability, the MWM experiment was carried out. Each animal performed four consecutive trials per day for 5 consecutive days in its 61-67 days of life. Before being gently released into the water at one of four starting location which was randomly selected, each individual rat was placed on the platform for $30 \mathrm{~s}$. Between trials the animals were allowed to rest for $30 \mathrm{~s}$. Recording was automatically terminated as escape latency when the animal found the platform and reached the platform. If rats could not find the platform within $60 \mathrm{~s}$, they were guided to the platform by hand and allowed to remain there for the equal time, and their escape latency was accepted as $60 \mathrm{~s}$. The mean latency of four trails each day was measured for individual animals every day.

The day after the acquisition phase, a probe test was conducted by removing the platform. Rats were allowed to swim freely in the pool for $60 \mathrm{~s}$. The time spent in the target quadrant which had previously contained the hidden platform was recorded to indicate the degree of memory consolidation.

\subsection{Morphometric analysis of dendritic spine density}

The Golgi-Cox staining method, which is a well-known method to stain whole neuron dendrites and spines in vivo ${ }^{20}$ and the method has been used in our study, ${ }^{21}$ was used to process the brains of rats. In brief, the brains were first stored in the dark for two days $\left(37^{\circ} \mathrm{C}\right)$ in Golgi-Cox solution, and then were sectioned at a thickness of $200 \mu \mathrm{m}$ in $6 \%$ sucrose with a vibratome (VT1000S, Leica, Germany). The coronal sections containing hippocampal dentate gyrus (DG) neurons were used in the present study. We collected one out of every 3 sections and got 4-5 sections per animal. Those sections were mounted to $2 \%$ gelatin-coated slides and stained with ammonia for $60 \mathrm{~min}$,

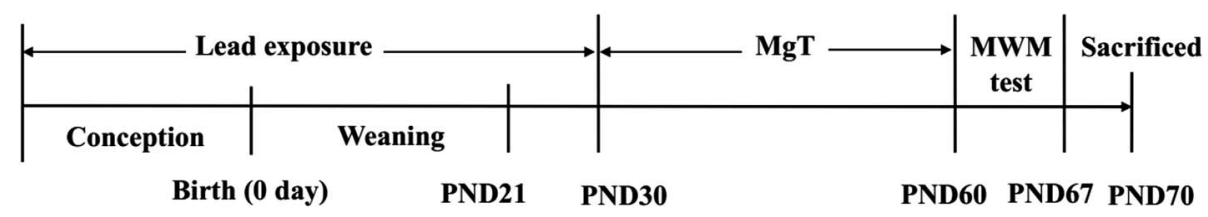

Fig. 1 Scheme of experiment design. 
washed with water three times, followed by Kodak Film Fix for $20 \mathrm{~min}$, and then washed with water, dehydrated, cleared, and mounted using a resinous medium. At last, DG neurons in hippocampus were imaged with a Nikon widefield microscope (Eclipse $80 i$, Nikon, Japan) by using a $40 \times$ objective. From all sections in each rat, about 6 neurons per section were chosen. Then, spine density (spine number per $10 \mu \mathrm{m}$ ) and dendrite number for each neuron were analyzed by sholl analysis using MATLAB software. The spines counted in the present study were on 2-3 stretches of the secondary dendrite about $20 \mu \mathrm{m}$ in length.

\subsection{Real-time quantitative PCR}

The total RNAs were extracted using the RNA kit (Axy-gen, Silicon Valley, USA) from the hippocampus DG homogenate of control and $\mathrm{Pb}$ rats with or without MgT treatment respectively ( $n=4$ for each group). Subsequently, First-Strand cDNA Synthesis SuperMix were used to complete the reverse transcription reaction according to the manufacturer's instructions (TransGene, Biotech), generating the first strand of total cDNA.

For real-time quantitative PCR (Q-PCR), the total reaction pool is $20 \mu \mathrm{L}$ which is composed of: $10 \mu \mathrm{L}$ of SYBR Green; $0.4 \mu \mathrm{L}$ of forward and reverse primer each; $2 \mu \mathrm{L}$ of cDNA template $(100$ ng $\mu \mathrm{L}^{-1}$ ) and $7.2 \mu \mathrm{L}$ of deionized water. The primers used in this protocol were listed as follows:

forward, 5'-GACGCAGCCGTCTTGAACTA- $3^{\prime}$ and

reverse, $5^{\prime}$-CAGCGCAATTCCATAGCCT- $3^{\prime}$ for NR2A,

forward, $5^{\prime}$-AATCAGAACGCCTCAACGC- $3^{\prime}$ and

reverse, $5^{\prime}$-GCTGACAATCTCAAGTCGGTAG-3' for GluR1,

forward, $5^{\prime}$-CTGTGCTATGTTGCCCTAGACTTC- $3^{\prime}$ and

reverse, $5^{\prime}$-CATTGCCGATAGTGATGACCTG- $3^{\prime}$ for $\beta$-actin, respectively. The real-time fluorescence PCR was performed with Real-Time PCR System machine (LightCycler® 96, Roche). Data analysis was done by using the $C_{\mathrm{t}}$ method and results were normalized to $\beta$-actin in software by Real-Time PCR System machine.

\subsection{Western blot}

Samples of hippocampal DG homogenate from four groups, control, MgT, $\mathrm{Pb}$ and $\mathrm{Pb}+\mathrm{MgT}$, were obtained, then the protein concentration was determined by using the bicinchoninic acid (BCA) method and equal amount of samples were resolved on polyacrylamide gels. Protein was then transferred to PVDF membrane and incubated in 5\% non-fat dry milk, after that protein was probed with such primary antibody as antiNR2A(\#4205S Cell Signaling), anti-NR2B(\#5580 Cell Signaling), anti-GluR1(\#8850 Cell Signaling), anti-PSD-95(Abcam 2723), and anti- $\beta$-actin(Abcam 16039) overnight followed by the appropriate HRP-coupled secondary antibody. Visualization of immunoreactive bands was detected by enhanced chemiluminescence and captured on chemiluminescence imaging instrument (GE ImageQuant LAS4000mini). For the western blot analyses, digital images were quantified using ImageJ software. The integrated optical density (IOD) of each immunoreactive band was measured. IOD was normalized to the IOD of $\beta$-actin band also in the same lane.

\subsection{Statistical analysis}

All data were expressed as mean \pm SEM. Statistical analysis was performed in GraphPad Prism 6.0 (GraphPad Software, Inc., La Jolla, CA). Statistical comparisons were made using One-way repeated ANOVA or Two-way repeated ANOVA when appropriate. A value of $p<0.05$ was considered to be statistically significant.

\section{Results}

\subsection{MgT rescues spatial memory deficits by chronic $\mathbf{P b}$ exposure}

Chronic $\mathrm{Pb}$ exposure is implicated in the impairment of learning and memory ${ }^{\mathbf{1 , 2 2}}$ and elevation of brain magnesium enhances the cognitive capacity and synaptic plasticity. ${ }^{19}$ To determine whether MgT rescues the cognitive capacity impaired by chronic $\mathrm{Pb}$ exposure, we tested rats for hippocampusdependent spatial memory using the MWM experiments. All rats showed a progressive reduction of the average latency to find the hidden platform during the 5 training days (Fig. 3A). In addition, four groups of rats had no significant differences at the swimming speed between any two groups (Fig. 3B). So MgT has no learning effects on $\mathrm{Pb}$ rats.

On day 6, we tested the spatial memory by the probe trial. Compared with $\mathrm{Pb}$-exposed rats, MgT treatment significantly increased the time spent in the target quadrant (Fig. $3 \mathrm{C}, \mathrm{Pb}$, $11.11 \pm 1.58, \mathrm{~Pb}+\mathrm{MgT}, 18.1 \pm 1.46, p<0.001)$ and reduced the latency to first entry to correct quadrant (Fig. $3 \mathrm{D}, \mathrm{Pb}, 14.8 \pm 2.5$, $\mathrm{Pb}+\mathrm{MgT}, 9.60 \pm 1.02, p<0.001)$. So, MgT can rescue the spatial memory deficits resulting from chronic $\mathrm{Pb}$ exposure during development. In addition, consistent with previous study (Slutsky, et al., 2010), MgT also enhanced the spatial memory in control rats (Fig. 3C, Control, $16.30 \pm 1.42$; MgT, $20.63 \pm 1.26, p$ $<0.05$ ) and reduced the latency to first entry to correct quadrant (Fig. 3D, Control, $12.96 \pm 1.09 ; \mathrm{MgT} 9.69 \pm 0.92, p<0.05$ ).

\subsection{MgT increases spine density and dendrite number of tertiary branch order in hippocampal DG area in Pb-exposed rats}

Exposure to $\mathrm{Pb}$ in rats during the critical window for rodent nervous system development leads to decreased spine density ${ }^{21}$ and loss of synapses, disrupts synaptic signaling and thus leads to progressive loss of cognition. ${ }^{23}$ Slutsky et al. ${ }^{19}$ demonstrates that increase in brain $\mathrm{Mg}^{2+}$ leads to increase in functional connectivity, synaptic plasticity, and enhancement of learning and memory. To determine whether MgT increases spine density in Pb-exposed rat, Golgi-Cox staining method was performed. Golgi-Cox impregnated dendritic arborization and dendritic spines were showed in Fig. 4A and representative sections of Golgi-Cox stained dendrites of granule cell in hippocampus dentate gyrus were showed in Fig. 4B. Pb-exposed with MgT-treated rats increased the number of dendritic spine in hippocampal DG area by $53.6 \%$ compared with Pb-exposed rats $(p<0.001$, Fig. $4 \mathrm{C})$. Consistent with our recent study, ${ }^{21}$ the number of dendritic spine in $\mathrm{Pb}$-exposed rats exhibited $10.33 \%$ decrease $(p<0.001)$ compared with control group. In 

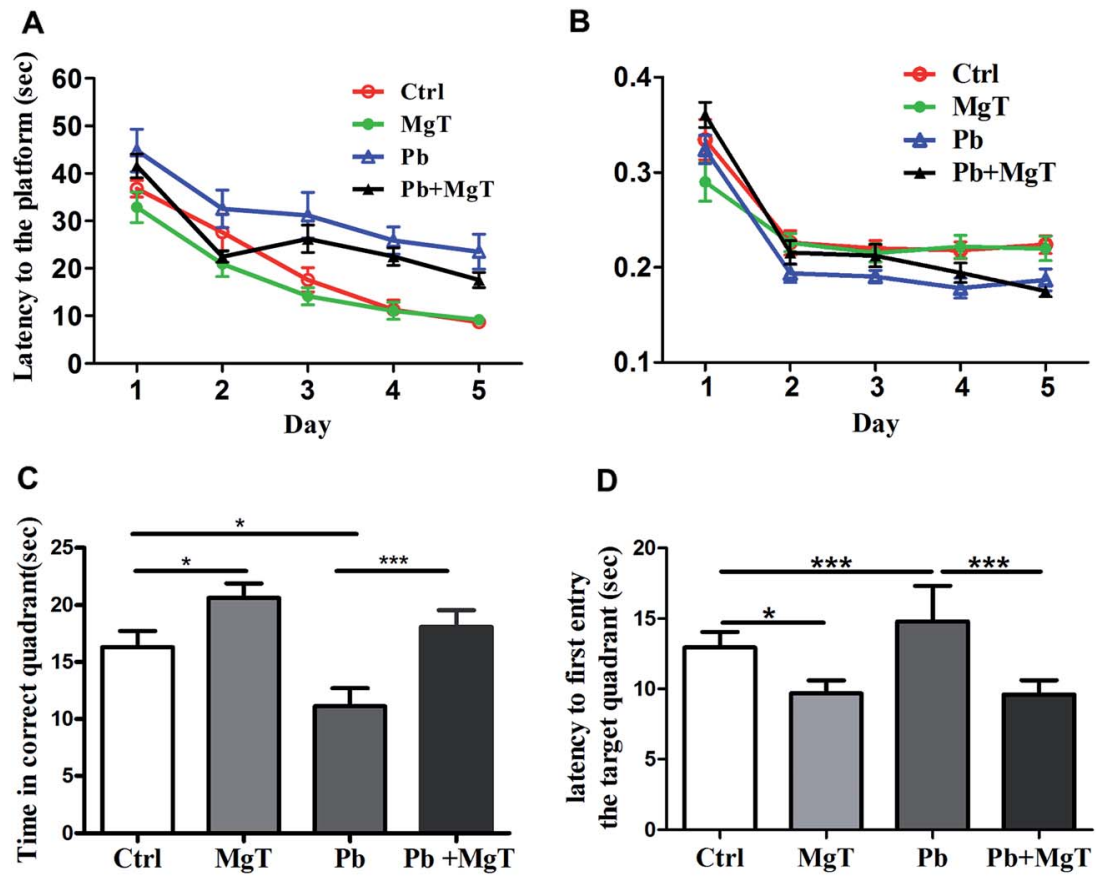

D

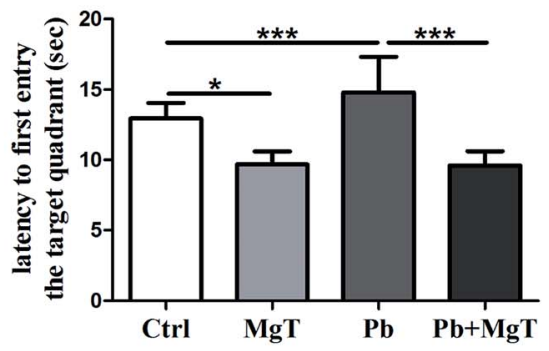

E
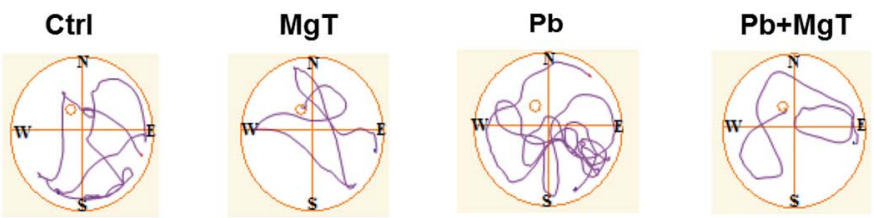

Fig. 3 MgT rescues spatial memory deficits in Pb-exposed rats. (A) Mean escape latencies to find the hidden platform for each of the four groups during the water maze training trials. (B) Swimming speed of the four groups during the water maze training trials. (C) Time spent in the correct quadrant and (D) latency to first entry to the target quadrant of the four groups in the probe trial on the sixth day. (E) Representative swimming paths of the four groups in probe test of the MWM experiment. The directions "North", "South", "East", and "West" are indicated as "N", "S", "E", and "W", respectively. The "North-West" quadrant was the target quadrant. There were 11, 11, 9, 9 male rats in Control, MgT, Pb and MgT + Pb, respectively. $(* p<0.05, * * * p<0.001)$.

addition, the number of dendritic spine increased about $14.4 \%$ in MgT-treated rats compared with control group $(p<0.001)$.

Moreover, as shown in Fig. 4D, Pb-exposed with MgT-treated rats significantly increased the dendrite number of tertiary branch order compared with $\mathrm{Pb}$-exposed rats $(\mathrm{Pb}, 2.26 \pm 0.34 ; \mathrm{Pb}$ $+\mathrm{MgT}, 5.08 \pm 0.37, p<0.001)$ and MgT induced a significant increasein dendrite number of tertiary branch order compared with control rats (control, $2.90 \pm 0.39 ; \operatorname{MgT} 4.43 \pm 0.48, p<0.05$ ). No obvious changes were observed in the dendrite number at primary branch and secondary branch order (Fig. 4D).

Taken together, MgT can increase spine density and dendrite number of tertiary branch order in hippocampal DG area in Pbexposed rats which may in turn influence the neuronal connection and the whole network in hippocampus, thus resulting in rescued spatial memory.

\subsection{Effect of $\mathrm{MgT}$ on the expression of some synaptic proteins in $\mathrm{Pb}$-exposed rats}

Dendritic spines are the locus of the vast majority of excitatory glutamatergic synapses where NMDARs, $\alpha$-amino-3-hydroxy5-methyl-4-isoxazolepropionic acid receptors (AMPARs) and
PSD-95 are critical constituents. ${ }^{24}$ Given that MgT increases spine density in $\mathrm{Pb}$-exposed rats is real, we wondered whether MgT could increase the expression of some synaptic proteins in $\mathrm{Pb}$-exposed rats. Here we checked the protein levels of NR2A, NR2B, PSD-95 and GluR1 in hippocampal DG area.

MgT significantly increased NR2A proteins in Pb-exposed rats $(p<0.05$, Fig. $5 \mathrm{~A}$ and B), but had no effect on NR2B proteins in Pb-exposed rats (Fig. 5A and C). Consistent with previous study, ${ }^{4,25}$ chronic $\mathrm{Pb}$ exposure significantly decreased the protein level of NR2A ( $p<0.05$, Fig. $5 \mathrm{~A}$ and B) without altering NR2B subunits (Fig. 5A and C). In addition, MgT significantly increased NR2A and NR2B proteins relative to control respectively ( $p<0.01$, Fig. 5B; $p<0.05$, Fig. 5C).

As a molecular organizer, PSD-95 not only anchors NMDARs to the postsynaptic membrane but also clusters GluR1containing AMPARs at glutamatergic synapses. Thus, based on the effect of MgT on the expression of NR2A, NR2B, we inferred that there should be an associated alteration in the level of PSD-95 and GluR1. The data showed that MgT elavated GluR1 proteins expression in $\mathrm{Pb}$-exposed rats (Fig. 5A and D, $p<0.05)$ without altering PSD-95 proteins in Pb-exposed rats 


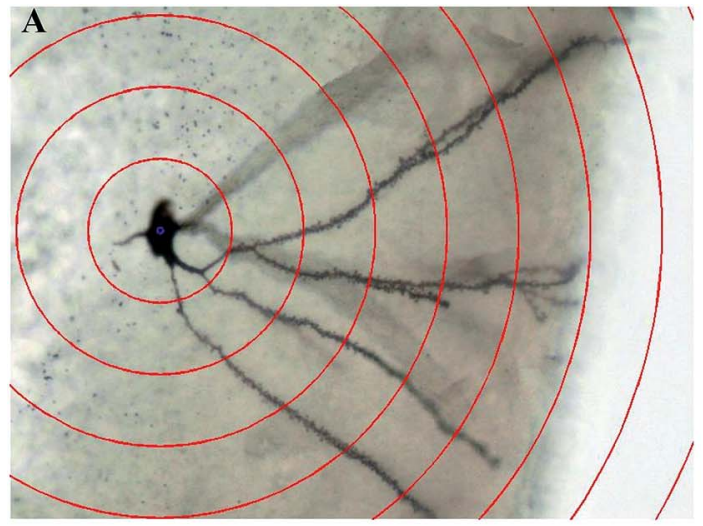

C

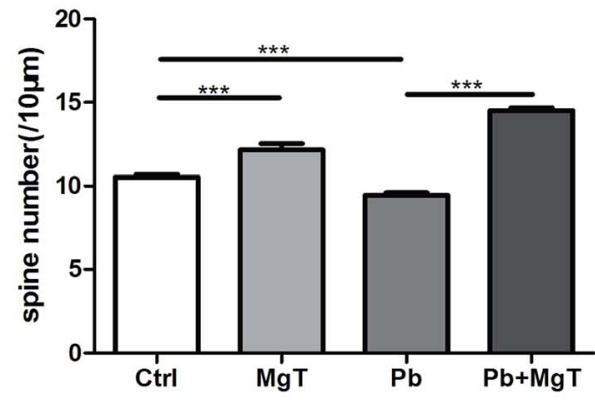

\section{B}

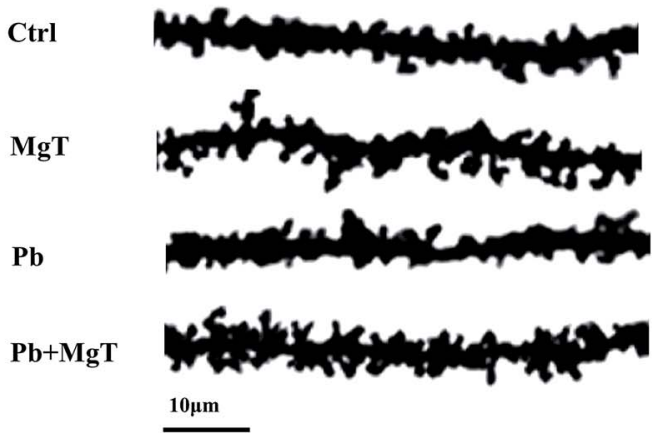

D

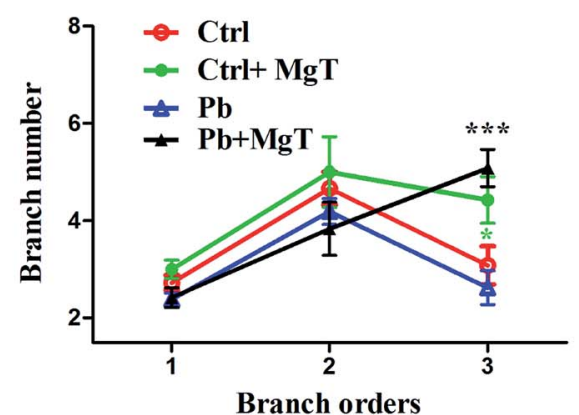

Fig. 4 MgT increases spine density and dendrite number of tertiary branch order on hippocampal DG area in Pb-exposed rats. (A) Representative images of Golgi-Cox impregnated granulosa cell in hippocampus dentate gyrus measured by Sholl analysis. (B) Representative sections of GolgiCox stained dendrites of granulosa cell in hippocampus dentate gyrus in four groups: control, $\mathrm{MgT}, \mathrm{Pb}$ (lead) and Pb $+\mathrm{MgT}$ (scale bar $=10 \mu \mathrm{m})(\mathrm{C})$ histograms plot showing the alteration of dendritic spine density (spines/10 $\mu \mathrm{m}$ ) after lead exposure or/and MgT treatment. (D) Quantification of dendrite number at different branch order in DG neurons of SD rats $(* * * p<0.001)$.

(Fig. 5A and E). Moreover, $\mathrm{Pb}$ exposure had no significant effect on expression of PSD-95 proteins (Fig. 5A and E) and GluR1 proteins (Fig. 5A and D), and MgT increased them obviously in control rats (Fig. 5D and $\mathrm{E}, p<0.05$ ).

These results indicated that NR2A and GluR1 play a critical role in $\mathrm{MgT}$ induced alterations in dendritic spine density impaired by chronic $\mathrm{Pb}$ exposure.

Further we examined the levels of NR2A and GluR1 mRNA in hippocampal DG area using Q-PCR. There showed significantly increased NR2A mRNA level in Pb-exposed with MgT-treated rats compared with $\mathrm{Pb}$-exposed rats $(p<0.001$, Fig. 6A). In addition, $\mathrm{Pb}$ decreased NR2A mRNA expression significantly ( $p$ $<0.001$ ) and MgT increased the expression of NR2A mRNA in control rats significantly ( $p<0.01)$.

MgT did not change GluR1 mRNA level in Pb-exposed rats (Fig. 6B). Meanwhile, MgT did not increase the expression of GluR1 mRNA significantly in control rats and $\mathrm{Pb}$ did not show obvious effect on this expression.

Taken together, these results indicated that MgT increased the expression of NR2A at the transcriptional and translational level while it increased GluR1 expression only at translational level.

\subsection{Pb concentration in hippocampus}

Finally, we examined $\mathrm{Pb}$ concentration within the hippocampus. In Table $1, \mathrm{~Pb}$ concentration in hippocampus of control and Pb-exposed rats on PND30 and four groups on PND70 are listed. There are significant difference in $\mathrm{Pb}$ concentration between control and $\mathrm{Pb}$-exposed group $(p<0.05)$ on PND30. On the PND70, there is no significant difference between control group and MgT group, and the same result between the $\mathrm{Pb}$ group and $\mathrm{Pb}+\mathrm{MgT}$ group. Compared with control rats, the $\mathrm{Pb}$ group and $\mathrm{Pb}+\mathrm{MgT}$ group have significant difference in hippocampal $\mathrm{Pb}$ concentration, respectively $(p<$ 0.001). These results indicate that $\mathrm{Pb}$-exposed rats had much higher hippocampal $\mathrm{Pb}$ concentration than control rats and $\mathrm{MgT}$ can not inhibit the fixation of $\mathrm{Pb}$ in the brain, that is, MgT did not exhibit chelating effect on $\mathrm{Pb}$.

\section{Discussion}

In this study, our results show that MgT can rescue the spatial memory deficits resulting from $\mathrm{Pb}$ exposure during development.

The cognitive function of rats exposed to $\mathrm{Pb}$ in "developmental window" are impaired and this effects can persist into adulthood. ${ }^{1}$ Based on this study, we exposed the rats with $\mathrm{Pb}$ during gestation and lactation until PND30. When rats grew up to young adult (60-70 days of age), their spatial memory were checked and hippocampal $\mathrm{Pb}$ concentration were measured. Consist with previous study, ${ }^{1}$ though $\mathrm{Pb}$ intake were abolished 

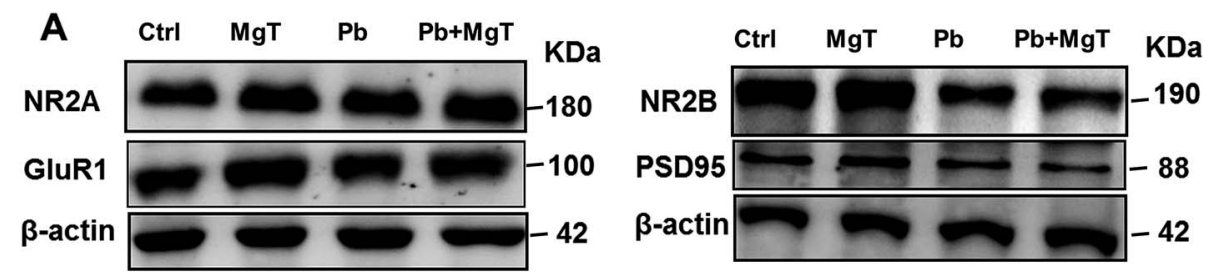

B

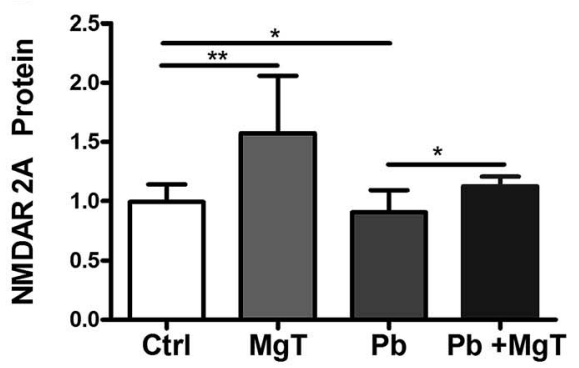

D

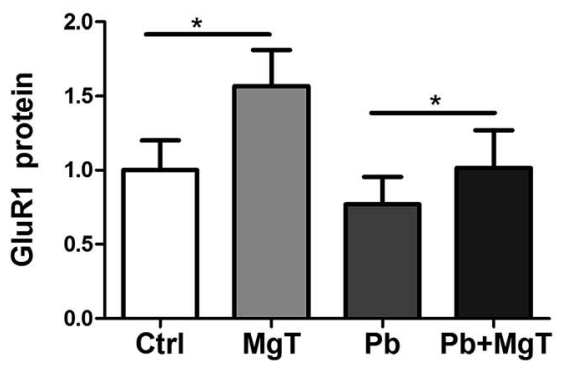

C

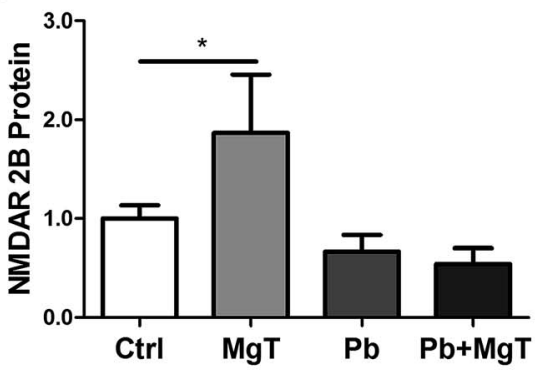

E

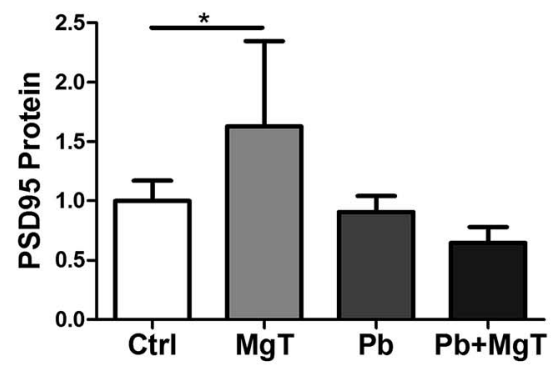

Fig. 5 Effects of MgT on protein expression of NR2A, NR2B, GluR1 and PSD-95 in hippocampal DG region in control and Pb-exposed rats. Representative immunoblot (A) and corresponding densitometric analysis showed relative expression of NR2A (B), NR2B (C), GluR1 (D) and PSD95 (E) in four groups: control, $\mathrm{MgT}, \mathrm{Pb}$ and $\mathrm{Pb}+\mathrm{MgT}$ respectively. Blot images were cropped for comparison. $\beta$-Actin was used as a loading control $(* p<0.05, * * p<0.01)$.

from PND31 to young adult, the impairment of the cognitive function also existed.

There are many drugs used for treating chronic $\mathrm{Pb}$-induced deficits early, especially the learning and memory. These drugs are common chelating agents, such as triethylene tetramine and penicillamine which exhibited partly treating effect on the $\mathrm{Pb}$ neurotoxicity, nevertheless, they showed many adverse effects at the same time. ${ }^{26,27}$ Latest animal studies ${ }^{28}$ show that some chemical substances, such as clioquinol and vitamin $\mathrm{B}_{12}$, can reverse the learning impairments induced by chronic $\mathrm{Pb}$ exposure in behavioral and electrophysiological experiment, but the action mechanisms of which are not been
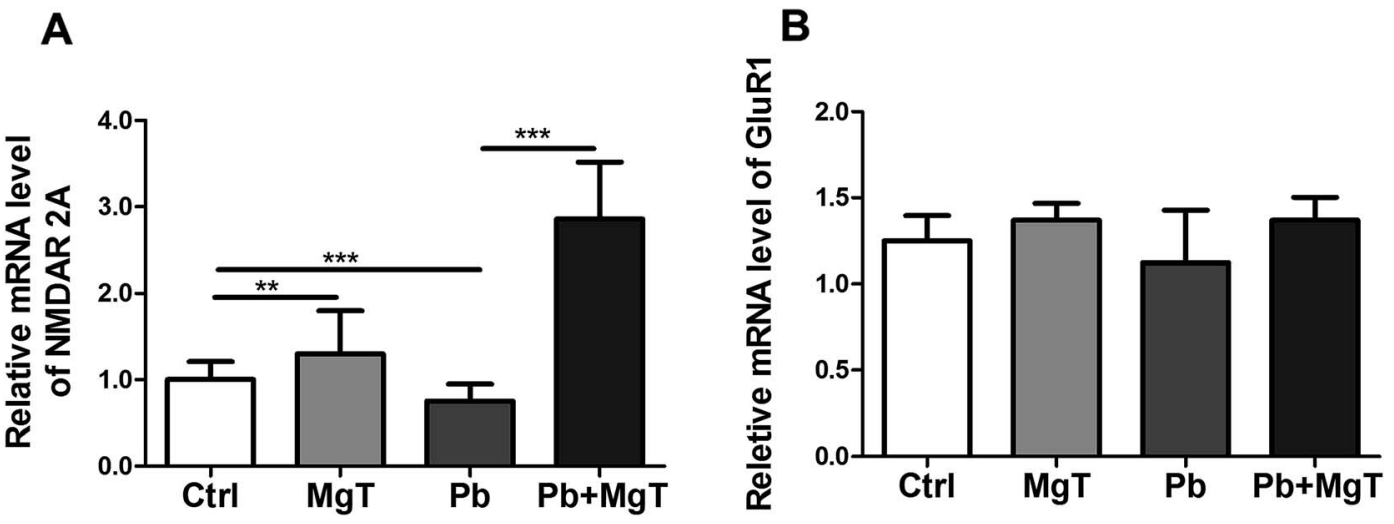

Fig. 6 Effect of MgT on mRNA levels of NR2A and GluR1 in hippocampal DG region in four groups. (A) mRNA levels of NR2A in four groups, (** $p<$ $0.01, * * * p<0.001)$. (B) mRNA levels of GluR1 in four groups, there were no statistical significance between any two groups of the four groups. The results were calculated as averages of triplicate experiments. 
Table $1 \mathrm{~Pb}$ concentrations in hippocampus $\left(\mu \mathrm{g} \mathrm{g}^{-1}\right)$

\begin{tabular}{|c|c|c|c|c|}
\hline & Control $(n=4)$ & Control $+\operatorname{MgT}(n=4)$ & Pb-exposed $(n=4)$ & $\mathrm{Pb}+\operatorname{MgT}(n=4)$ \\
\hline PND30 & $0.0459 \pm 0.147$ & & $0.812 \pm 0.147^{a}$ & \\
\hline PND70 & $0.0852 \pm 0.024$ & $0.0731 \pm 0.079$ & $0.713 \pm 0.11^{b}$ & $0.557 \pm 0.133^{b}$ \\
\hline
\end{tabular}

studied further. Besides, environmental enrichment have been used by researchers to reverse learning impairments in the cognitive function induced by $\mathrm{Pb}$. Though the reversal effect on the cognitive function was obvious, the mechanism of that has yet to be defined. ${ }^{10,29}$

In current study, MgT treatment for 30 days improved rats spatial memory in control group, which is consistent with study by Slucky et al. ${ }^{19}$ While the important finding is the improvement effect of MgT on spatial memory on Pb-exposed rats.

Dendritic spines are post-synaptic locations of excitatory synapses, receiving and processing synaptic information, which protrude from the shaft or branch of the dendrite. ${ }^{24}$ Loss of synapses disrupts synaptic signaling and thus leads to progressive loss of cognition. ${ }^{23}$ We have previously shown that dendritic spine density decreased in hippocampal CA1 area in $\mathrm{Pb}$-exposed rats on postnatal day 14 and postnatal day $21 .^{21}$ This decreased effect still exists on rat postnatal day 70 though $\mathrm{Pb}$ had been removed from the drinking water on postnatal day 30 . We firstly used MgT on Pb-exposed rats and found that MgT reversed the decrease of dendritic spine density and dendrite number mediated by chronic $\mathrm{Pb}$ exposure. And these changes are consistent with the changes in memory ability exhibited in MWM experiment. Thus we conclude that the rescue effect of MgT on memory ability impaired by $\mathrm{Pb}$ is partly for their reversed effect on spine density decreased by $\mathrm{Pb}$ at least.

As post-synaptic terminals, dendritic spines are the locus of the vast majority of excitatory glutamatergic synapses containing NMDARs and AMPARs. ${ }^{24}$ Functional NMDARs are necessary for spatial learning and memory, as assessed by the MWM. ${ }^{30}$ Though exposure to $\mathrm{Pb}$ impaired cognitive function and hampered the expression of glutamate receptors, the underlying mechanisms remain elusive. ${ }^{4,22}$ As one of main glutamate receptors, the NMDARs are composed of an obligatory NR1 subunit and one or more accessory subunits from the NR2 or NR3 families. NR2A and NR2B are the most abundant NR2 family members in the hippocampus. ${ }^{31-33}$ Several lines of studies show that chronic developmental $\mathrm{Pb}$ exposure results in decreased NR2A content in the hippocampus while NR2B mRNA levels either remain unchanged or exhibit a slight increase in Pb-exposed rats. ${ }^{\mathbf{4 , 2 5 , 3 4}}$ Consistent with these studies, our study further confirmed these changes of NMDAR subunits expression affected by $\mathrm{Pb}$. In this study, we showed that chronic dietary intake of $\mathrm{MgT}$ reversed NR2A protein and mRNA expression levels by chronic $\mathrm{Pb}$ exposure. And the reverse effect is consistent with the rescue effect of MgT on memory ability and on spine density. Meanwhile MgT increased NR2A protein and mRNA levels in hippocampal DG subregions in control rats, but there are two studies ${ }^{\mathbf{1 8 , 1 9}}$ which exhibited that NR2A protein did not change by MgT, the reason should be the different region studied.

As for NR2B we have the same result with Abumaria, N., et al. that MgT increased NR2B expression in control rats. ${ }^{18,19}$ But considering the two contradictive results that $\mathrm{MgT}$ increase NR2B expression and spatial learning ability and that $\mathrm{Pb}$ exposure result in NR2B mRNA levels either remained unchanged or exhibited a slight increase $e^{4,25,34,35}$ and decrease spatial learning ability, we can not explain their relationship to our knowledge. In our study MgT have not exhibited significant effect on NR2B expression in Pb-exposed rats, but there is a decreasing trend which maybe suggest a changeover effect on $\mathrm{Pb}$.

AMPARs are tetramers comprised of four glutamate receptor subunits, GluR1-GluR4. Accumulated evidence has demonstrated that synaptic insertion of GluR1-containing AMPARs contributes to the synaptic potentiation during LTP, experiencedependent neuronal plasticity as well as learning and memory. ${ }^{36-38}$ By examining the levels of GluR1 protein expression in the hippocampus of aged animals, many studies have shown the role of GluR1-containing AMPARs in agingassociated cognitive deficits. To our surprise, the results of these studies are contradictory, for some studies report that GluR1 expression in the hippocampus is age-related decrease or increase, while others show no significant difference between young adult and aged rodents. ${ }^{39-41}$ Using in situ hybridization, Guilarte et al. have found that there is no change of GluR1 mRNA in Pb-exposed animals at 50 days of age, ${ }^{10}$ likewise we cannot find the mRNA change in hippocampus DG region in $\mathrm{Pb}$ exposed rats though there is a decreased trend. But on protein level, MgT treatment increased GluR1 in control and $\mathrm{Pb}$ exposed animals. Thus if only taking GluR1 protein expression level in account, MgT exhibited the rescue effect. Of course, what we tested is the total GluR1 protein level including the protein intracellular and inserting in cell membrane, and only inserting into postsynaptic membrane can it play a part in synaptic transduction. That is, to declare the relationship between GluR1 and synaptic plasticity, the expression on postsynaptic membrane of GluR1 protein level should be tested.

As a major component of PSD, PSD-95 is a central postsynaptic scaffold and signaling hub that promotes formation and maturation of dendritic spines. ${ }^{42}$ PSD-95 is dispensable for synaptic anchoring NMDARs to the postsynaptic membrane and facilitates clustering of GluR1-containing AMPARs at glutamatergic synapses on both excitatory and inhibitory neurons. Besides these, PSD-95 controls the different physiological roles played by the NMDAR complex. ${ }^{43} \mathrm{~Pb}$ exposure did not affected PSD-95 protein expression which is consistent with a previous 
study. ${ }^{10}$ MgT significantly increased PSD-95 protein level in control but not in $\mathrm{Pb}$-exposed rats.

In summary, we show that MgT is important in rescuing memory ability impairment by $\mathrm{Pb}$. By reversing the expression of such synaptic proteins as NR2A and GluR1 decreased by $\mathrm{Pb}$, MgT increases the number of dendritic spines and thus the number of synapse which is benefit to improve memory ability deficits. Because MgT does not exhibit chelating effect on $\mathrm{Pb}$ and $\mathrm{Mg}^{2+}$ can serve as a cofactor of enzymes, participating in the process of intracellular signal transduction, the possible mechanism how MgT rescues impaired memory ability by $\mathrm{Pb}$ may be that $\mathrm{Mg}^{2+}$ promotes some enzymes activity decreased by $\mathrm{Pb}$ by elevating brain $\mathrm{Mg}^{2+}$. Of course the mechanism should be explored further. Our study suggest that MgT can be a potential complementary agent in the treatment of $\mathrm{Pb}$ intoxication.

\section{Conflict of interests}

The authors declare no competing financial interests.

\section{Acknowledgements}

We thank professor Guosong Liu for providing reagents (Magnesium-L-threonate powder) in support of these studies. This work was supported by the National Key Basic Research Program of China (973 Program, No. 2012CB525003), the National Science Foundation of China (No. 21477031, 31200851, 31401671), the Program for New Century Excellent Talents in University (NCET-12-0835), Specialized Research Fund for the Doctoral Program of Higher Education (No. 20130111110024), Key Laboratory of Xin'an Medicine Ministry of Education, Anhui University of Chinese Medicine (No. 2015sbgj005) and the Huangshan Young Scholar Fund of Hefei University of Technology (407-037030).

\section{References}

1 A. C. Kuhlmann, J. L. Mcglothan and T. R. Guilarte, Developmental lead exposure causes spatial learning deficits in adult rats, Neurosci. Lett., 1997, 233(233), 101-104.

2 P. Mushak, et al., Prenatal and postnatal effects of low-level lead exposure: integrated summary of a report to the U.S. Congress on childhood lead poisoning, Environ. Res., 1989, 50(1), 11-36.

3 G. Winneke, H. Lilienthal and U. Krämer, The Neurobehavioural Toxicology and Teratology of Lead, Arch. Toxicol., 1996, 18(95), 57-70.

4 M. K. Nihei, et al., $N$-methyl-D-aspartate receptor subunit changes are associated with lead-induced deficits of longterm potentiation and spatial learning, Neuroscience, 2000, 99(2), 233-242.

5 D. Y. Ruan, et al., Impairment of long-term potentiation and paired-pulse facilitation in rat hippocampal dentate gyrus following developmental lead exposure in vivo, Brain Res., 1998, 806(2), 196-201.
$6 \mathrm{~J}$. Q. She, et al., Effect of ganglioside on synaptic plasticity of hippocampus in lead-exposed rats in vivo, Brain Res., 2005, 1060(1-2), 162-169.

7 D. M. Zhu, et al., Protection by a taurine supplemented diet from lead-induced deficits of long-term potentiation/ depotentiation in dentate gyrus of rats in vivo, Neuroscience, 2005, 134(1), 215-224.

8 H. J. Carlisle and M. B. Kennedy, Spine architecture and synaptic plasticity, Trends Neurosci., 2005, 28(4), 182-187.

9 M. M. Ge, et al., Wnt/ $\beta$-catenin Signaling Involved in Protective Effect of EGCG on Lead-induced Impairments of Spine Formation in Rat Hippocampus, RSC Adv., 2015, 5(40), 31622-31628.

10 T. R. Guilarte, et al., Environmental enrichment reverses cognitive and molecular deficits induced by developmental lead exposure, Ann. Neurol., 2003, 53(1), 50-56.

$11 \mathrm{~J}$. H. Koenig and K. Ikeda, Synaptic vesicles have two distinct recycling pathways, J. Cell Biol., 1996, 135(3), 797-808.

12 L. Nowak, et al., Magnesium gates glutamate-activated channels in mouse central neurones, Nature, 1984, 307(5950), 462-465.

13 M. L. Mayer, G. L. Westbrook and P. B. Guthrie, Voltagedependent block by $\mathrm{Mg}^{2+}$ of NMDA responses in spinal cord neurones, Nature, 1984, 309(5965), 261-263.

$14 \mathrm{H}$. Murck, Magnesium and affective disorders, Nutr. Neurosci., 2002, 5(6), 375-389.

15 T. Miyashita, et al., $\mathrm{Mg}(2+)$ block of Drosophila NMDA receptors is required for long-term memory formation and CREB-dependent gene expression, Neuron, 2012, 74(5), 887-898.

16 E. I. Moser, et al., Impaired Spatial Learning after Saturation of Long-Term Potentiation, Science, 1998, 281(5385), 2038-2042.

17 I. Slutsky, et al., Enhancement of Synaptic Plasticity through Chronically Reduced $\mathrm{Ca}^{2+}$ Flux during Uncorrelated Activity, Neuron, 2004, 44(5), 835-849.

18 N. Abumaria, et al., Effects of elevation of brain magnesium on fear conditioning, fear extinction, and synaptic plasticity in the infralimbic prefrontal cortex and lateral amygdala, J. Neurosci., 2011, 31(42), 14871-14881.

19 I. Slutsky, et al., Enhancement of Learning and Memory by Elevating Brain Magnesium, Neuron, 2010, 65(2), 165-177.

20 R. Gibb, B. Kolb, R. Gibb and B. Kolb, A method for vibratome sectioning of Golgi-Cox stained whole rat brain, J. Neurosci. Methods, 1998, 79(1), 1-4.

$21 \mathrm{~F}$. $\mathrm{Hu}$, et al., Developmental lead exposure alters synaptogenesis through inhibiting canonical Wnt pathway in vivo and in vitro, PLoS One, 2014, 9(7), e101894.

22 C. D. Toscano, et al., Developmental Pb exposure alters NMDAR subtypes and reduces CREB phosphorylation in the rat brain, Dev. Brain Res., 2002, 139, 217-226.

23 P. Penzes, et al., Dendritic spine pathology in neuropsychiatric disorders, Nat. Neurosci., 2011, 14(3), 285-293.

24 O. V. B. U. Halbach, Structure and function of dendritic spines within the hippocampus, Ann. Anat., 2009, 191(6), 518-531. 
25 M. K. Nihei and T. R. Guilarte, NMDAR-2A subunit protein expression is reduced in the hippocampus of rats exposed to $\mathrm{Pb}^{2+}$ during development, Mol. Brain Res., 1999, 66(1-2), 42-49.

26 M. E. Mortensen and P. D. Walson, Chelation therapy for childhood lead poisoning. The changing scene in the 1990s, Clin. Pediatr., 1993, 32(5), 284-291.

27 S. Porru and L. Alessio, The use of chelating agents in occupational lead poisoning, Occup. Med., 1996, 46(1), 4148.

28 W. H. Chen, et al., Clioquinol and vitamin B12 (cobalamin) synergistically rescue the lead-induced impairments of synaptic plasticity in hippocampal dentate gyrus area of the anesthetized rats in vivo, Neuroscience, 2007, 147(3), 853-864.

29 X. Cao, S. Huang and D. Ruan, Enriched environment restores impaired hippocampal long-term potentiation and water maze performance induced by developmental lead exposure in rats, Dev. Psychobiol., 2008, 50(3), 307-313.

30 R. G. M. Morris, et al., Selective impairment of learning and blockade of long-term potentiation by an $N$-methyl-Daspartate receptor antagonist, AP5, Nature, 1986, 319(6056), 774-776.

$31 \mathrm{H}$. Monyer, et al., Heteromeric NMDA receptors: molecular and functional distinction of subtypes, Science, 1992, 256(5060), 1217-1221.

$32 \mathrm{H}$. Monyer, et al., Developmental and regional expression in the rat brain and functional properties of four NMDA receptors, Neuron, 1994, 12(3), 529-540.

33 M. Masu, et al., Molecular Characterization of NMDA and Metabotropic Glutamate Receptors, Ann. N. Y. Acad. Sci., 1993, 707, 153-164.

34 T. R. Guilarte and J. L. Mcglothan, Hippocampal NMDA receptor mRNA undergoes subunit specific changes during developmental lead exposure, Brain Res., 1998, 790(790), 98-107.

35 X. Y. Zhang, et al., Effect of developmental lead exposure on the expression of specific NMDA receptor subunit mRNAs in the hippocampus of neonatal rats by digoxigenin-labeled in situ hybridization histochemistry, Neurotoxicol. Teratol., 2002, 24(2), 149-160.

36 V. A. Derkach, et al., Regulatory mechanism of AMPA receptors in synaptic plasticity, Nat. Rev. Neurosci., 2007, 8(2), 101-113.

37 Y. Hayashi, et al., Driving AMPA receptors into synapses by LTP and CaMKII: requirement for GluR1 and PDZ domain interaction, Science, 2000, 287(5461), 2262-2267.

38 D. Mitsushima, et al., Contextual learning requires synaptic AMPA receptor delivery in the hippocampus, Proc. Natl. Acad. Sci. U. S. A., 2011, 108(30), 12503-12508.

39 N. Andres, et al., Increase in AMPA receptors in aged memory-impaired rats is not associated with increase in monoamine oxidase B levels, Neuroscience, 2000, 101(4), 807-810.

40 M. M. Adams, et al., Caloric restriction and age affect synaptic proteins in hippocampal CA3 and spatial learning ability, Exp. Neurol., 2008, 211(1), 141-149.

41 B. Selvakumar, R. L. Huganir and S. H. Snyder, Snitrosylation of stargazin regulates surface expression of AMPA-glutamate neurotransmitter receptors, Proc. Natl. Acad. Sci. U. S. A., 2009, 106(38), 16440-16445.

42 E. D. El-Husseini, et al., PSD-95 involvement in maturation of excitatory synapses, Science, 2000, 290(5495), 1364-1368.

43 T. Tezuka, et al., PSD-95 promotes Fyn-mediated tyrosine phosphorylation of the $N$-methyl-D-aspartate receptor subunit NR2A, Proc. Natl. Acad. Sci. U. S. A., 1999, 96(2), 435-440. 